\title{
Review
}

\section{Humor and Laughter May Influence Health: III. Laughter and Health Outcomes}

\author{
Mary Payne Bennett ${ }^{1}$ and Cecile Lengacher ${ }^{2}$ \\ ${ }^{1}$ Indiana State University College of Nursing and ${ }^{2}$ University of South Florida
}

This is part three of a four-part series reviewing the evidence on how humor influences physiological and psychological well-being. The first article included basic background information, definitions and a review of the theoretical underpinnings for this area of research. The second article discussed use of humor as a complementary therapy within various clinical samples, as well as evidence concerning how a sense of humor influences physiological and psychological wellbeing. This third article examines how laughter influences health outcomes; including muscle tension, cardio-respiratory functioning and various stress physiology measures.

Keywords: humor - health - laughter - physiology

\section{Introduction}

As discussed in the first two parts of this series, there has been some evidence that sense of humor and exposure to a humorous stimulus can influence specific health outcomes $(1,2)$. However, more conclusive evidence for possible physiological effects of humor is documented in studies that examine the actions of the natural outcome of humor-mirthful laughter. If humor has a role in physical healing, one might expect some measurable physical changes in response to the action of laughing. Over the years, several studies have been conducted to document various physiological outcomes related to laughter. Some of these investigations were conducted during the infant stages of psychoneuroimmunology theory, with little subsequent comment or replication effort. Most researchers in this area have noted that it is very difficult to obtain funding for humor studies, particularly those involving expensive physiological outcome measures. In addition, it should be noted that most of the early studies used all male samples, and all of the studies have small sample sizes. Given these limitations,

For reprints and all correspondence: Mary Payne Bennett, Indiana State University College of Nursing, 749 Chestnut Street, Terre Haute, IN 47809. E-mail: mbennett2@isugw.indstate.edu we have outlined what is known about the physiological effects of laughter.

\section{Laughter as Exercise}

Many lay publications and websites claim that laughter is equivalent to some forms of aerobic exercise. A quick search of the Internet using the Google search engine, using the terms laughter and aerobic exercise turned up the following statements all on the first page of the search: 'Laughter may have similar effects on the body as aerobic exercise.... increasing the blood flow in the same way as a bout of aerobic exercise... Laughter stimulates heart and blood circulation and is equivalent to any other standard aerobic exercise. But are any of these statements based on scientific research? The truth is, documentation supporting these assertions is very limited.

One very early report by Paskind et al. (3) examined the impact of laughter upon muscle tone. Unlike the effects of some exercise, it was demonstrated that periods of intense laughter lead to decreased skeletal muscle tone or relaxation of muscle groups. During laughter, various muscle groups are activated for periods of seconds at a time, while the period immediately after the laugh leads to general muscle relaxation. This post-laughter relaxation can last up to $45 \mathrm{~min}$ (3). 
A more recent attempt at documenting the effect of laughter on muscles used a more sophisticated technique (4). Based on Paskind's earlier work that indicated a period of muscular relaxation follows laughter, this study examined how laughter and several other respiratory movements influenced spinal motor excitability, as measured by the Hoffmann reflex (H-reflex). The H-reflex is a clinical method of measurement, similar to tapping the patella to elicit a deep tendon reflex. However, the H-Reflex is different from a tendon tap in two respects. First, the stimulus is an electrical shock to sensory fibers coming from muscle spindles rather than a mechanical stretching of those receptors. Second, the response is recorded using an electromyographic (EMG). The H-Reflex method is a preferred measurement because the stimulus is better controlled, the response can be measured more precisely, and changes in muscle stretch receptor sensitivity do not affect the results. When looking at the $\mathrm{H}$-reflex, increased twitching indicates increased spinal cord excitability. Thirteen healthy volunteers doing a variety of 10 different tasks (including laughter, simulated laughter and various respiratory movements) participated in this study. The results indicated that laughter resulted in H-reflex suppression. H-reflex percentage differed between the neutral task $(79.4 \pm 16.1 \%)$, true laughter $(43.7 \pm 17.9 \%)$ and simulated laughter $(66.6 \pm 24.3 \%)$. Coughing also resulted in H-reflex suppression, but not as deeply as true laughter. During the other respiratory maneuvers, the H-reflex increased compared to the neutral task. Findings from this study indicate that both laughter and simulated laughter decreased spinal motor excitability, and that true laughter evoked more H-reflex depression than simulated laughter, which suggests that mirth on its own can depress the H-reflex, leading to the post-laughter muscle relaxation response noted in Paskind's earlier work.

In addition to changes in muscle tone, there is evidence that laughter leads to changes in respiratory function. Fry's work demonstrates that laughter leads to episodes of sharply sporadic deep breathing (5). However, these breathing changes do not appear to significantly improve oxygen saturation levels, at least in healthy individuals (6). Of course, with healthy subjects, their normal oxygen saturation rate would be expected to be near or at $100 \%$. It may be that there is little to no room for improvement in this group. It would be interesting to learn how laughter influences oxygen saturation rates in a more compromised population, such as persons with chronic lung disease.

Fry's later work supports the theory that laughter can lead to acute changes in cardio-respiratory functioning. Hard laughter leads to increased heart-rate, respiratory rate and oxygen consumption, which is indeed similar to changes seen with aerobic exercise. However, these changes are in effect only during periods of intense laughter, which are very difficult to sustain for any length of time (7). This makes it unlikely that laughter can be regarded as a realistic substitute for aerobic exercise.

\section{Laughter, Stress and Anxiety}

According to James-Lange theory of emotion and later theories (8), all intense emotions, regardless of their content, lead to activation of the sympathetic nervous system. This was tested in an investigation by Averill, in which three groups of men were exposed to either a humorous movie, a sad movie or a neutral movie (9). Physiological responses were determined by heart rate, blood pressure, respiratory rate, skin temperature and galvanic skin response. Both the humorous stimulus and the sad stimulus produced significant increases in galvanic skin response (GSR), indicating activation of the sympathetic nervous system. However, while exposure to a sad stimulus caused significant increases in blood pressure over the control group, the blood pressure of those exposed to humor remained stable, even though the GSR reading of both groups would indicate activation of the sympathetic nervous system (9). The results of this study suggest that while a humorous stimulus can activate the sympathetic nervous system similar to some stressors, it may also help buffer some of the negative effects of sympathetic activation upon blood pressure.

To determine if humor can be beneficial in the treatment of anxiety, the impact of a humorous stimulus upon anxiety and heart rate was investigated during a stressful waiting period (10). A sample of 53 college students was told that they would receive a shock after a brief waiting period. Subjects in the experimental group listened to a humorous tape while waiting to receive a shock, the placebo control group listened to a nonhumorous tape during this waiting period, and nonintervention control group did not listen to a tape. Subjects in the humor group reported decreased anxiety during the anticipatory period $(P<0.05)$, while those with the highest level of sense of humor (measured by SHRQ) had the lowest reported anxiety $(P<0.01)$. However, the heart rate of the three groups were not significantly different, and all three groups experienced increased heart rate during the waiting period $(P<0.001)$. The authors concluded that the effect of humor may be primarily cognitive, rather than having a physical stress reduction capability, because heart rate was not significantly different among the groups (10). However, as the investigations reported above indicate, laughter tends to increase heart and respiratory rate in itself, and therefore heart-rate may not have been the best physiological measure of reduced anxiety for this experiment. Also, it would have been interesting to see what results this intervention had on GSR's and blood pressure, as it 
appears that activation of the sympathetic nervous system was involved, as in Averill's study (9).

Research examining how laughter influences stress hormones is somewhat conflicting. One early study looked at the results of viewing four different films on urinary excretion of epinephrine and norepinephrine in 20 female subjects (11). The four films were chosen to elicit different emotions. The first was a natural-scenery film, which was expected to be very bland and not to elicit any strong emotions. A second film was funny, and elicited mirth and laughter. A third film was a war movie and was used to elicit feelings of tragedy and sadness. The fourth film was a horror story chosen to elicit anxiety. All of the subjects viewed each film and served as their own control mechanism in a pre-post-test design. Urinary hormone levels were measured during a $90 \mathrm{~min}$ control period before each film, during each film, and finally during a $90 \mathrm{~min}$ post-film session. Urinary epinephrine levels decreased significantly during the natural-scenery film $(P<0.01)$. Urinary epinephrine levels increased significantly during the war film $(P<0.05)$. Urinary epinephrine and norepinephrine both increased significantly during the humorous film $(P<0.05)$. Urinary epinephrine increased the most dramatically during the anxiety provoking film $(P<0.05)$, and norepinephrine levels also increased significantly during this film $(P<0.001)$, see Fig. $1(11)$.

The authors concluded that there is a correlation between increased urinary excretion of epinephrine and strong emotional arousal, independent of the type of emotion being elicited. However, the lack of a control group, the use of an all female subject group, and the methodology involving the subjects all viewing the films in the same order, are weaknesses in this study. The results obtained could be due to the order of the films, as the first film lead to the lowest levels of hormone excretion, while the last film lead to the highest levels.

Building on this earlier work, a more recent project examined the effect of viewing a humorous film on a wide variety of stress hormones, using direct testing of hormone levels in serum (12). Subjects in the treatment group viewed a humorous video, while those in control

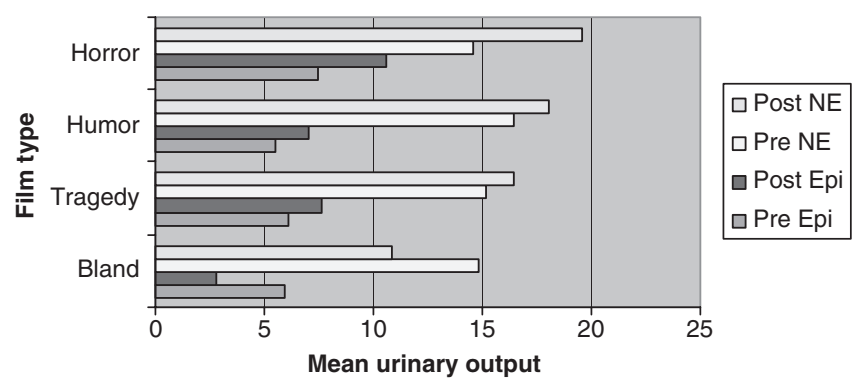

Figure 1. Histogram represents hormonal response to viewing four different film types by healthy women. Bars indicate mean urinary output of epinephrine and norepinephrine pre- and post-stimulus (11). group were kept in a quiet room for the same amount of time. Endocrine testing was performed pre, during and post stimuli. The results demonstrated that serum cortisol $(P=0.011)$, growth hormone $(P=0.0005)$ and plasma dopac - a metabolite of dopamine $(P=0.025)$, all decreased with laughter. There was no significant change in serum prolactin, beta-endorphins, plasma epinephrine or plasma norepinephrine in either the experimental or the control group. The control group demonstrated no significant changes in any of the endocrine measures used. The authors concluded that laughter decreases stress hormones, and could theoretically act to buffer the effects of stress on the immune system. Because cortisol is immunosuppressive, the authors theorized that laughter could act as an immunoenhancer by decreasing levels of this particular stress hormone $(12,13)$. This study, while controlled, used a rather small sample $(n=10)$ and included male subjects only. It should also be noted that while some media releases have claimed that laughter makes people feel good because it increases beta-endorphins, this study did not support this proposed result.

In summary, the materials reviewed in this section support that the act of laughter can lead to immediate increases in heart rate, respiratory rate, respiratory depth and oxygen consumption. These increases are then followed by a period of muscle relaxation, with a corresponding decrease in heart rate, respiratory rate and blood pressure. The effect of laughter on the so called 'stress hormones' of epinephrine, norepinephrine and cortisol is equivocal, with the few studies conducted thus far demonstrating some conflicting results. This is important because it is theorized that if laughter does, in fact, decrease stress hormones, this would be one mechanism that might explain the proposed connection between laughter and immune function, and from there to improved health outcomes. The investigation into the effects of laughter on the endocrine system and the immune system is clearly an area in which additional research is needed. The next article in this series will review the evidence available thus far concerning how laughter influences various immune system components.

\section{References}

1. Bennett MP, Lengacher CA. Humor and laughter may influence health. I. History and background. Evid Based Complement Alternat Med 2006;3:61-3.

2. Bennett MP, Lengacher CA. Humor and laughter may influence health. II Complementary therapies and humor in a clinical population. Evid Based Complement Alternat Med 2006;3:187-90.

3. Paskind J. Effects of laughter on muscle tone. Arch Neurol Psychiatry 1932;28:623-8.

4. Overeem S, Taal W, Ocal Gezici E, Lammers G, Van Dijk J. Is motor inhibition during laughter due to emotional or respiratory influences? Psychophysiology 2004;41:254-8. 
5. Fry W. The respiratory components of mirthful laughter. J Biol Psychol 1977;19:39-50.

6. Fry W. Mirth and oxygen saturation levels of peripheral blood. Psychother Psychosom 1971;19:76-84.

7. Fry W, Savin W. Mirthful laughter and blood pressure. Humor: Int J Humor Res 1988;1:49-62.

8. Mandler G. Emotion. In: Brown R, Galanter E, Hess E, Mandler G (eds). New Directions in Psychology. New York: Holt, 1962, 267-343.

9. Averill J. Autonomic response patterns during sadness and mirth. Psychophysiology 1969;5:399-414.

10. Yovetich N, Dale A, Hudak M. Benefits of humor in reduction of threat-induced anxiety. Psychol Rep 1990;66:51-8.
11. Levi L. The urinary output of adrenalin and noradrenalin during pleasant and unpleasant emotional states. Psychosom Med 1965;27:403-19.

12. Berk L, Tan S, Napier B, Evy W. Eustress of mirthful laughter modifies natural killer cell activity. Clin Res 1989; 37:115A.

13. Berk L, Tan S, Nehlsen-Cannarella S, Napier B, Lewis J, Lee J, et al. Humor associated laughter decreases cortisol and increases spontaneous lymphocyte blastogenesis. Clin Res 1988; $36: 435 \mathrm{~A}$.

Received January 23, 2007; accepted February 16, 2007 


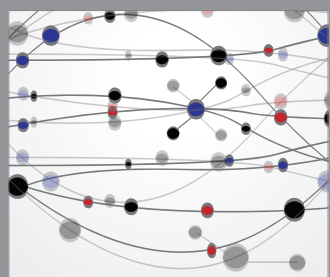

The Scientific World Journal
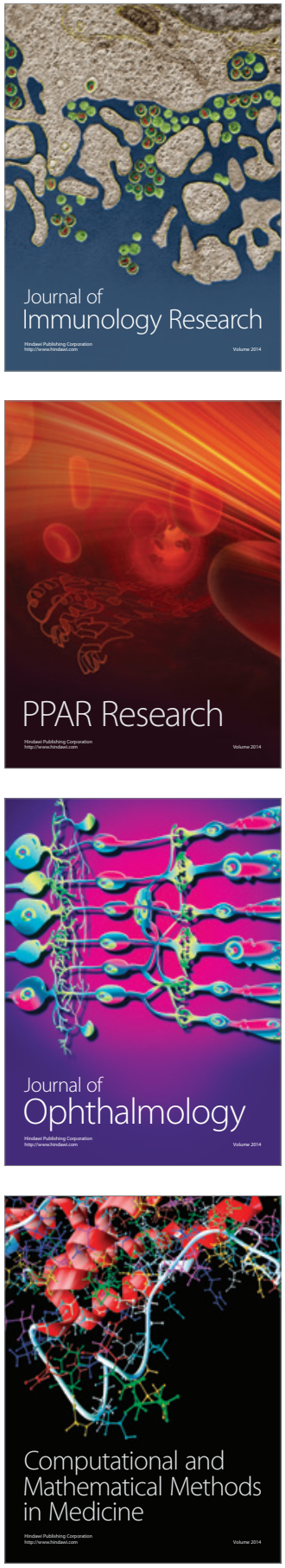

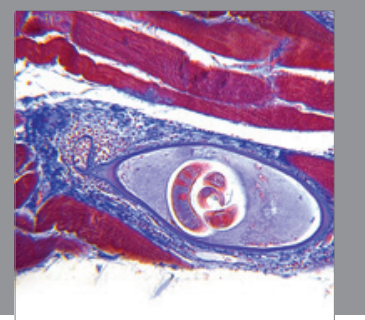

Gastroenterology

Research and Practice
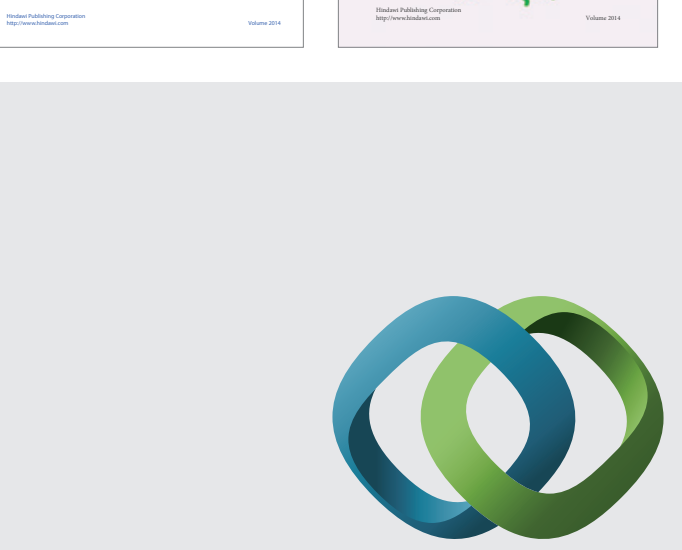

\section{Hindawi}

Submit your manuscripts at

http://www.hindawi.com
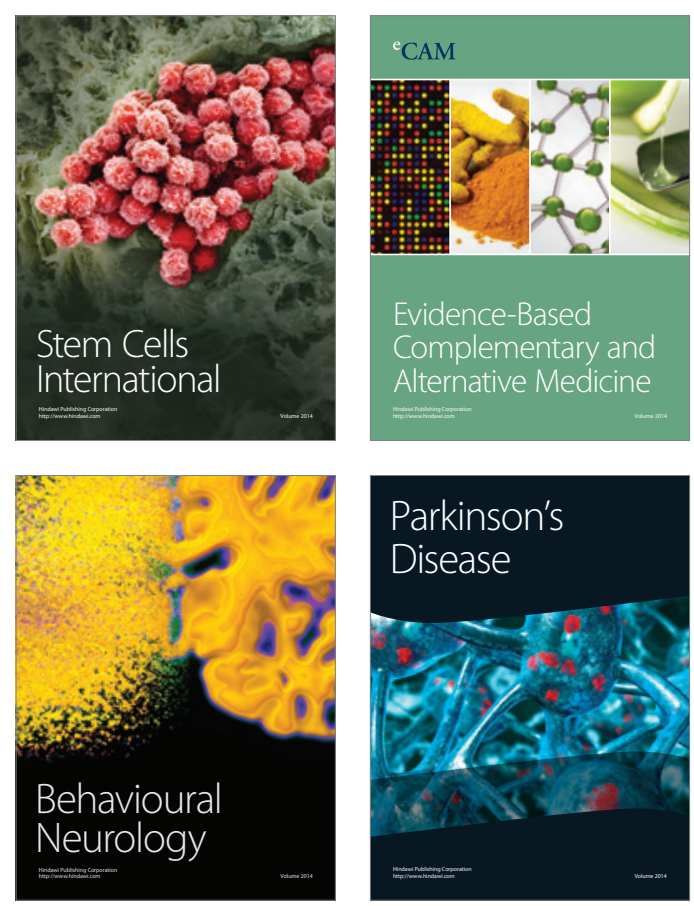

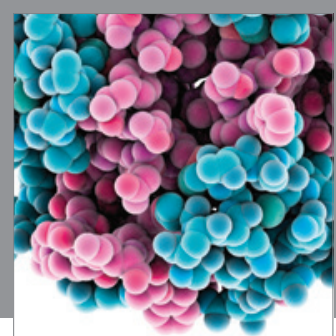

Journal of
Diabetes Research

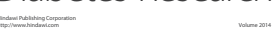

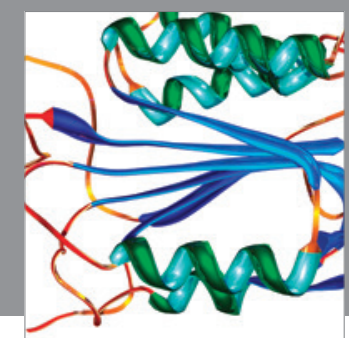

Disease Markers
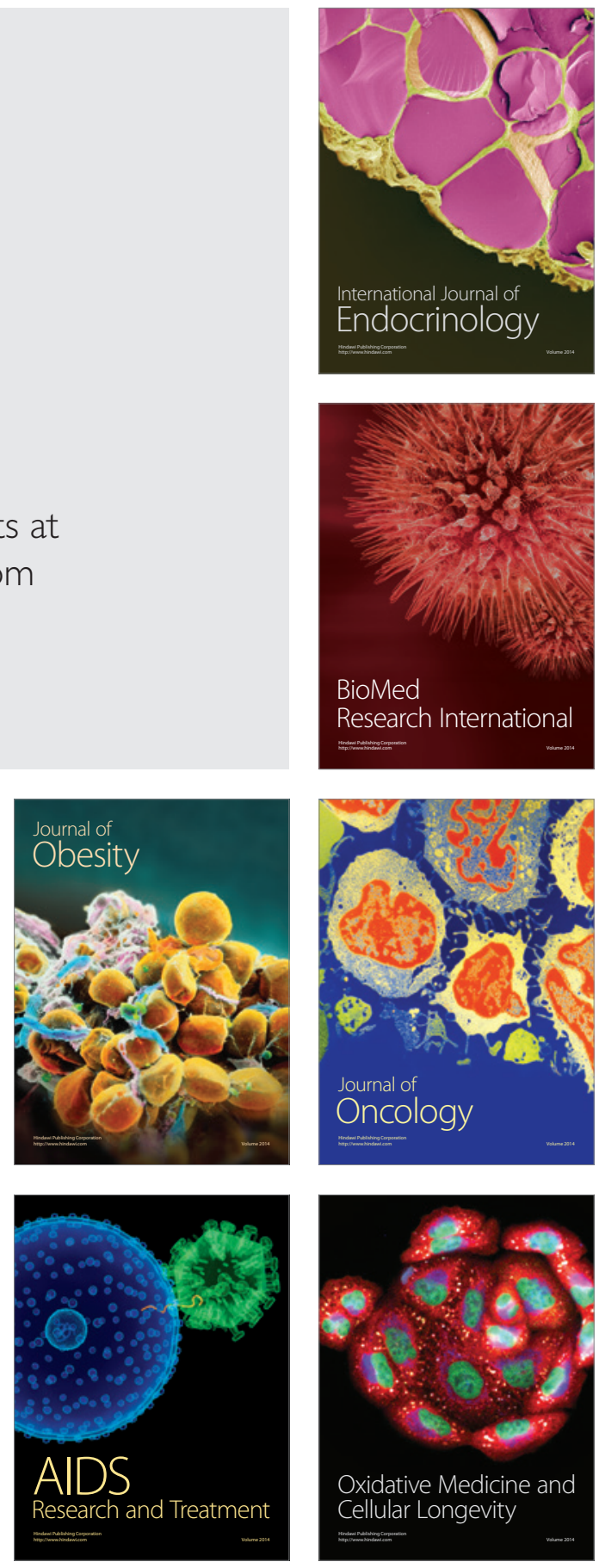